\title{
Accurate Maximum Power Point Tracking Algorithm Based on a Photovoltaic Device Model
}

\author{
Lhoussain El Bahir and Touria Hassboun \\ Laboratory of Electrical Engineering and Control Systems (LGECOS), National School of Applied Sciences, Cadi Ayyad University, \\ Av. Abdelkrim El Khattabi B.P. 575, 40000 Marrakech, Morocco
}

Correspondence should be addressed to Lhoussain El Bahir; 1.elbahir@uca.ma

Received 24 March 2017; Accepted 5 July 2017; Published 10 August 2017

Academic Editor: Urs Aeberhard

Copyright ( 2017 Lhoussain El Bahir and Touria Hassboun. This is an open access article distributed under the Creative Commons Attribution License, which permits unrestricted use, distribution, and reproduction in any medium, provided the original work is properly cited.

\begin{abstract}
An accurate method is proposed to track the maximum power point of a photovoltaic module. The method is based on the analytical value of the maximum power point voltage, determined from a mathematical model of the photovoltaic panel. The method has the advantage of accuracy without any oscillations, as with certain conventional methods. The algorithm has also the ability to track accurately the maximum power point under variable atmospheric conditions and load changes. Experimental results are presented to show the effectiveness of the method. The implementation of the method needs an online measurement of irradiance, panel temperature, and panel current and voltage.
\end{abstract}

\section{Introduction}

The solar energy is one of the most competitive sources of electrical energy, especially photovoltaic (PV) devices. PV panels are devices that convert solar energy into electricity in a clean way and offer an alternative solution of electric power generation in all industry sectors and domestic applications, particularly in remote areas.

The PV device performances are certainly very attractive but could be influenced by some factors, especially the relative panel orientation to solar rays, measurement equipment, power converters, load and/or grid connection, and the extraction of the maximum electric power. An overall optimization of the system should be performed to achieve the best possible performance. A variety of methods to deal with these aspects has been proposed and studied in the literature $[1,2]$.

The maximum power point tracking techniques (MPPT) represent one of the most studied subjects in PV systems. Since the MPP varies with the temperature and irradiation levels, these techniques are equipped by specific algorithms to extract the maximum available electric power. The determined maximum power voltage is used as a reference voltage in the power converter loop. Many strategies to find the MPP are available in the literature for different equipment technologies, variable environmental conditions, complexity, cost, range of effectiveness, convergence speed, and accuracy tracking when irradiation and/or temperature change. An exhaustive review of different MPPT algorithms can be found in [3-6].

The P\&O (Perturb and Observe) and the Incremental Conductance algorithms are the most common [7-12]. Their main advantages are the easy implementation, low computational demand, and only the measured voltage and current are required as information about the PV array. But they also have some drawbacks, like oscillations around the MPP in steady-state conditions and poor tracking under rapidly changing irradiation levels. This is due to the fact that the search for maximum power point is related to the choice of the perturbation step. The latter infects the convergence time and the amplitude of the oscillations antagonistically.

The constant voltage (CV) is also a well-known method in which the operating voltage $V_{\max }$ is set to a fixed percentage of the open-circuit voltage $V_{\mathrm{oc}}$. The main problem with this algorithm is that $V_{\max }$ is not always at the fixed percentage of $V_{\mathrm{oc}}$ and the energy is wasted when 
the circuit is opened to measure $V_{\text {oc }}[4,5,13]$. Instead of operating with voltage, one can operate with current to obtain the so-called short-current pulse-based MPPT which is similar to the CV method. The operating current $I_{\max }$ is then a fixed percentage of the short-circuit current $I_{\mathrm{sc}}$. Many improved versions of these methods are published in the literature $[9,12]$.

Look-up table method and Curve-fitting-based MPPT are offline techniques [14]. In the first one, technical data and panel characteristics at different environmental conditions are stored in a memorized table. Then, measured power of the PV panel is compared with those of stored values to track MPP and operating point is shifted to the new maximum power point. Thus, the system requires a large memory capacity to store all necessary data for varying atmospheric conditions. This method is slow and not very accurate [5]. In the second method, the power-voltage characteristic of PV panels is approached by a polynomial function. The MPP voltage is deduced according to the polynomial coefficients. The operating point on the characteristics of the panel is then moved to track the MPP. This approach requires also large memory and large computation time to calculate the polynomial coefficients for different environmental conditions $[5,14]$.

In the so-called "beta $(\beta)$ method," the MPP is approximated using an intermediate variable $\beta$ which depends on the parameters, the current, and the voltage of the PV panel $[3,5]$. The variable $\beta$ is continuously computed and compared with a constant reference to track MPP. This method gives fast tracking speed during varying atmospheric conditions.

Some methods are based on neural networks and fuzzy $\operatorname{logic}[3,11,15,16]$. The mean advantage is their ability to take into account the nonlinearities without handling nonlinear mathematical models. However, a good user experience on the PV module characteristics is needed.

In this paper, an accurate method is proposed to track the maximum power point of a photovoltaic module. The method is based on a mathematical model of the PV module to compute the theoretical value of the MPP voltage, for an online measured PV current, PV voltage, irradiance, and temperature. The method has the advantage of accuracy without any oscillations, as with some previously mentioned methods. This accuracy depends of course on the accuracy of the model. The MPPT algorithm has also the ability to track, with a fast speed, the maximum power point under variable atmospheric conditions and load changes. Simulation and experimental tests were performed to validate the method.

The rest of the paper is organized as follows. In Section 2, the mathematical model of photovoltaic panel is presented. The proposed theoretical computation of the MPP voltage is introduced in Section 3. The steps of the deduced MPPT algorithm are described in Section 4. Section 5 deals with the implementation of the proposed MPPT method, including the validation of the model, the description of the experimental platform, and finally, the presentation and discussion of the obtained results. The conclusion is given in Section 6.

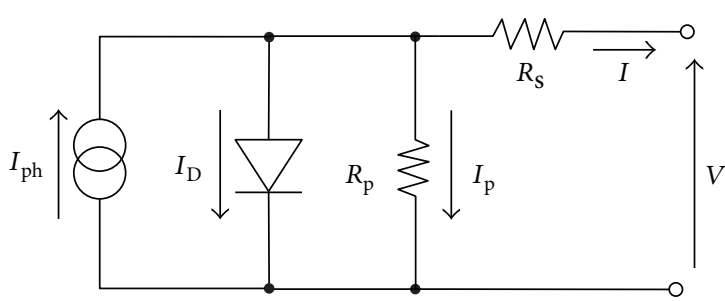

FIgURE 1: Equivalent circuit model of the ideal PV cell.

\section{Mathematical Model of Photovoltaic Panel}

A photovoltaic cell is an electric power generator. Under solar irradiation, it converts the absorbed photon energy into electrical energy. Depending on the load to which it is connected, the cell can behave as a current generator or a voltage generator. A PV system naturally exhibits nonlinear currentvoltage $(I-V)$ characteristic which depends upon the solar irradiation and cell temperature. The single-diode model with the equivalent circuit, as shown in Figure 1, is a simple model that is commonly used because of its practical convenience and the fact that it represents a reasonable compromise between accuracy and simplicity [17].

The circuit comprises a current source in parallel with a diode and a shunt resistor $R_{\mathrm{p}}$, materializing the leakage current at the junction, and a resistivity grid resistor $R_{\mathrm{s}}$. The current $I_{\mathrm{ph}}$ is generated by the incident light and is directly proportional to the sun irradiation, $I_{0}$ is the reverse saturation or leakage current of the diode, and $I_{\mathrm{D}}$ is the Shockley diode current. Based on the circuit of Figure 1, the mathematical model of a photovoltaic cell can be defined in accordance with

$$
\begin{aligned}
I & =I_{\mathrm{ph}}-I_{\mathrm{D}}-I_{\mathrm{p}} \text { with } I_{\mathrm{D}}=I_{0}\left(e^{V / a V_{\mathrm{t}}}-1\right), \\
I_{\mathrm{p}} & =\frac{V+R_{\mathrm{s}} I}{R_{\mathrm{p}}},
\end{aligned}
$$

where $V_{\mathrm{t}}=k T / q$ is the junction thermal voltage, $q$ is the electron charge, $k$ is the Boltzmann constant, $T$ is the temperature of the $p$ - $n$ junction, and $a$ is the diode ideality constant.

The relationship between $I$ and $V$ is given by

$$
I=I_{\mathrm{ph}}-I_{0}\left(e^{\left(V+R_{\mathrm{s}} I\right) / a V_{\mathrm{t}}}-1\right)-\frac{V+R_{\mathrm{s}} I}{R_{\mathrm{p}}} .
$$

The current $I_{\mathrm{ph}}$ describes the spectrum of the photovoltaic cell and depends on climatic conditions such as ambient temperature and irradiation $G$ as follows:

$$
I_{\mathrm{ph}}=\frac{G}{G_{\mathrm{r}}}\left[I_{\mathrm{scr}}-k_{\mathrm{i}}\left(T-T_{\mathrm{r}}\right)\right],
$$

where $T_{\mathrm{r}}$ is the reference temperature, $I_{\text {scr }}$ is the cell's shortcircuit current at $T_{\mathrm{r}}, k_{\mathrm{i}}$ is the temperature coefficient of the short circuit, and $G_{\mathrm{r}}$ is the nominal irradiation.

The diode saturation current $I_{0}$ depending on temperature may be expressed by

$$
I_{0}=I_{\mathrm{or}}\left(\frac{T}{T_{\mathrm{r}}}\right)^{3} e^{q E_{\mathrm{g}} / A k\left(\left(1 / T_{\mathrm{r}}\right)-(1 / T)\right)},
$$



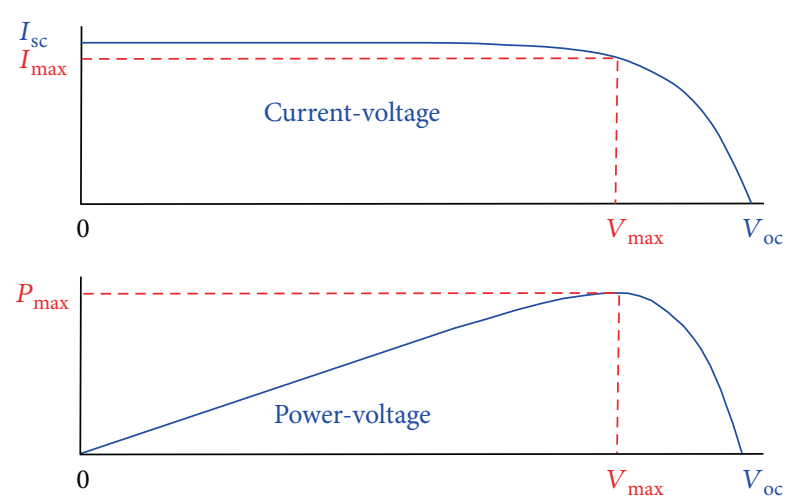

Figure 2: Current-voltage $(I-V)$ and power-voltage $(P-V)$ characteristics of a PV module.

where $I_{\mathrm{or}}$ is the nominal saturation current and $E_{\mathrm{g}}$ is the band gap energy.

Practical PV array is composed of several interconnected cells. The configuration of the PV array model requires the knowledge of the physical parameters of the panel, which are always provided with reference to the nominal conditions or at standard test conditions of temperature and solar irradiation. The most important parameters widely used for describing the cell electrical performance are the opencircuit voltage $V_{\mathrm{oc}}$, the short-circuit current $I_{\mathrm{scr}}$, the voltage at the maximum power point (MPP) $\left(V_{\max }\right)$, the current at the MPP $\left(I_{\max }\right)$, and the maximum experimental peak output power $\left(P_{\max }\right)$. The rest of parameters could be deduced from the mathematical model equations and the PV technology.

The model of a PV panel with $n_{\mathrm{p}}$ cells in parallel and $n_{\mathrm{s}}$ cells in series can be represented by (6) [17].

Let

$$
\begin{aligned}
R_{\mathrm{st}} & =\frac{n_{\mathrm{s}}}{n_{\mathrm{p}}} R_{\mathrm{s}}, \\
R_{\mathrm{pt}} & =\frac{n_{\mathrm{s}}}{n_{\mathrm{p}}} R_{\mathrm{p}}, \\
\xi & =1+\frac{R_{\mathrm{st}}}{R_{\mathrm{pt}}}, \\
\beta & =\frac{1}{V_{\mathrm{t}} a n_{\mathrm{s}}}, \\
h & =\left(I_{\mathrm{ph}}+I_{0}\right) n_{\mathrm{p}} .
\end{aligned}
$$

Then, (1) can be written as

$$
\xi I=h-I_{0} n_{\mathrm{p}} e^{\beta\left(V+R_{\mathrm{st}} I\right)}-\frac{V}{R_{\mathrm{pt}}} .
$$

\section{Theoretical Computation of the MPP Voltage}

As the PV power is given by $P=I V$, (6) can be rewritten as

$$
\xi P=h V-I_{0} n_{\mathrm{p}} V e^{\beta\left(V+R_{\mathrm{st}} P / V\right)}-\frac{V^{2}}{R_{\mathrm{pt}}} .
$$

The so known current-voltage $(I-V)$ and power-voltage $(P-V)$ characteristics are represented in Figure 2, for a given temperature $T$ and a given irradiance $G$.

The maximum power $P_{\max }$ of the PV module is obtained for the voltage $V_{\max }$ and the current $I_{\max }$. Due to the nonlinearity nature of (7), the determination of the analytical expression of the maximum power voltage $V_{\max }$ is not obvious by using the standard derivative computation. In the literature, a numerical approach is often used to solve this equation. In this paper, we showed that, by determining the analytical equation of the tangent at each point of the power-voltage $(P-V)$ curve, one can deduce that $V_{\max }$ is given by

$$
V_{\max }=\frac{\beta h\left(R_{\mathrm{st}}-R_{\mathrm{GL}}\right)+\xi-\left(R_{\mathrm{GL}} / R_{\mathrm{pt}}\right)}{\beta\left(\left(R_{\mathrm{st}} / R_{\mathrm{GL}}\right)-1\right)\left(\xi+\left(R_{\mathrm{GL}} / R_{\mathrm{pt}}\right)\right)},
$$

where $R_{\mathrm{GL}}$ represents the global load, seen by the PV panel (Figure 3).

Indeed, consider (6) and suppose that we move from a point $\left(I_{n}, V_{n}\right)$, for a sampling index $n$, of the $I-V$ curve to a closely neighboring point $\left(I_{n+1}, V_{n+1}\right)$, indexed by $n+1$, as illustrated in Figure 4, in such way that

$$
\begin{aligned}
I_{n+1} & =I_{n}-i, \\
V_{n+1} & =V_{n}+v,
\end{aligned}
$$

where $i$ is a very small variation of the current $(i \rightarrow 0)$ and $v$ is the corresponding very small variation of the voltage $(v \rightarrow 0)$.

From (6), one can write for both points:

$$
\begin{gathered}
\xi I_{n}=h-I_{0} n_{\mathrm{p}} e^{\beta\left(V_{n}+R_{\mathrm{st}} I_{n}\right)}-\frac{V_{n}}{R_{\mathrm{pt}}}, \\
\xi I_{n+1}=h-I_{0} n_{\mathrm{p}} e^{\beta\left(V_{n+1}+R_{\mathrm{st}} I_{n+1}\right)}-\frac{V_{n+1}}{R_{\mathrm{pt}}} .
\end{gathered}
$$

By taking into account (9) in the second equation of (10), we have

$$
\xi I_{n}-\xi i=h-I_{0} n_{\mathrm{p}} e^{\beta\left(V_{n}+R_{\mathrm{st}} I_{n}+v-R_{\mathrm{st}} i\right)}-\frac{V_{n}+v}{R_{\mathrm{pt}}}
$$

and then

$$
\xi I_{n}-\xi i=h-I_{0} n_{\mathrm{p}} e^{\beta\left(V_{n}+R_{\mathrm{st}} I_{n}\right)} e^{\beta\left(v-R_{\mathrm{si}} i\right)}-\frac{V_{n}+v}{R_{\mathrm{pt}}} .
$$

Since $i \rightarrow 0$ and $v \rightarrow 0$ as assumed before, we can consider that $\beta\left(v-R_{\mathrm{st}} i\right) \rightarrow 0$ and then make the following approximation:

$$
e^{\beta\left(v-R_{\mathrm{st}} i\right)} \simeq 1+\beta\left(v-R_{\mathrm{st}} i\right)
$$




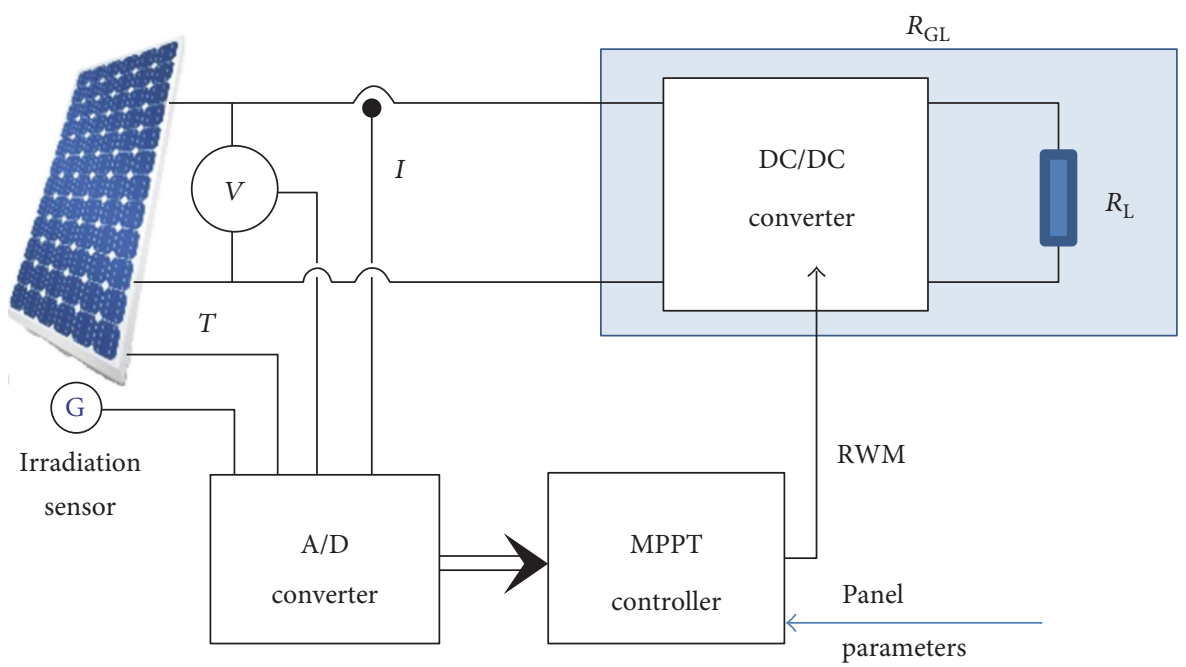

FIGURE 3: PV system with MPPT controller.

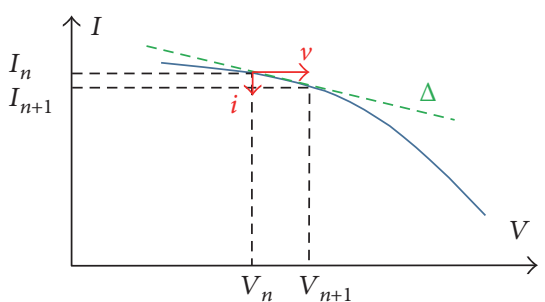

Figure 4: $I-V$ curve section.

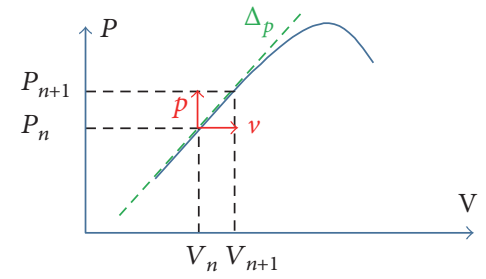

Figure 5: A power-voltage curve section.

Equation (12) can then be approximated as

$$
\begin{aligned}
\xi I_{n}-\xi i= & h-I_{0} n_{\mathrm{p}} e^{\beta\left(V_{n}+R_{\mathrm{st}} I_{n}\right)}\left[1+\beta\left(v-R_{\mathrm{st}} i\right)\right]-\frac{V_{n}+v}{R_{\mathrm{pt}}}, \\
\xi I_{n}-\xi i= & \underbrace{h-I_{0} n_{\mathrm{p}} e^{\beta\left(V_{n}+R_{\mathrm{st}} I_{n}\right)}-\frac{V_{n}}{R_{\mathrm{pt}}}}_{\xi I_{n}} \\
& -I_{0} n_{\mathrm{p}} e^{\beta\left(V_{n}+R_{\mathrm{st}} I_{n}\right)} \beta\left(v-R_{\mathrm{st}} i\right)-\frac{v}{R_{\mathrm{pt}}} .
\end{aligned}
$$

Eliminating $\xi I_{n}$ and its equivalent expression leads to

$$
\left(\xi+I_{0} n_{\mathrm{p}} e^{\beta\left(V_{n}+R_{\mathrm{st}} I_{n}\right)} \beta R_{\mathrm{st}}\right) i=\left(I_{0} n_{\mathrm{p}} e^{\beta\left(V_{n}+R_{\mathrm{st}} I_{n}\right)} \beta+\frac{1}{R_{\mathrm{pt}}}\right) v,
$$

and then

$$
i=\frac{I_{0} n_{\mathrm{p}} e^{\beta\left(V_{n}+R_{\mathrm{st}} I_{n}\right)} \beta+\left(1 / R_{\mathrm{pt}}\right)}{\xi+I_{0} n_{\mathrm{p}} e^{\beta\left(V_{n}+R_{\mathrm{st}} I_{n}\right)} \beta R_{\mathrm{st}}} v .
$$

According to (10), $I_{0} n_{\mathrm{p}} e^{\beta\left(V_{n}+R_{\mathrm{st}} I_{n}\right)}=-\xi I_{n}-\left(V_{n} / R_{\mathrm{pt}}\right)+h$. This allows us to write (16) as

$$
i=\frac{\left(h-\xi I_{n}-\left(V_{n} / R_{\mathrm{pt}}\right)\right) \beta+\left(1 / R_{\mathrm{pt}}\right)}{\xi+\left(h-\xi I_{n}-\left(V_{n} / R_{\mathrm{pt}}\right)\right) \beta R_{\mathrm{st}}} v .
$$

This expression seems better, numerically speaking, than (16) which contains an exponential term.

Equation (17) or (16) can be interpreted as the tangent $(\Delta)$ to the $I-V$ curve at the point $\left(I_{n}, V_{n}\right)$, Figure 4 .

Moving from the point $\left(I_{n}, V_{n}\right)$ to the point $\left(I_{n+1}, V_{n+1}\right)$ on the $I-V$ characteristic means also that the power moves from the point $\left(P_{n}, V_{n}\right)$ to the point $\left(P_{n+1}, V_{n+1}\right)$ (Figure 5) so that

$$
P_{n+1}=P_{n}+p,
$$

where $p$ is the power variation corresponding to the voltage variation $v$.

Equation (16) can be written as

$$
\begin{aligned}
V_{n+1} I_{n+1} & =V_{n} I_{n}+p \Rightarrow\left(V_{n}+v\right)\left(I_{n}-i\right) \\
& =V_{n} I_{n}+p \Rightarrow v I_{n}-v i-V_{n} i=p .
\end{aligned}
$$

We can consider that $i v$ could be neglected in (19) as $i \rightarrow 0$ and $v \rightarrow 0$. So,

$$
p \simeq v I_{n}-V_{n} i .
$$

Equations (20) and (17) lead to

$$
p=\left[I_{n}-V_{n}\left(\frac{\left(h-\xi I_{n}-\left(V_{n} / R_{\mathrm{pt}}\right)\right) \beta+\left(1 / R_{\mathrm{pt}}\right)}{\xi+\left(h-\xi I_{n}-\left(V_{n} / R_{\mathrm{pt}}\right)\right) \beta R_{\mathrm{st}}}\right)\right] v,
$$

which can be interpreted as the tangent $\left(\Delta_{p}\right)$ to the power curve at the point $\left(P_{n}, V_{n}\right)$ (Figure 5). We can deduce that 
when $P_{n}=P_{\max }$ and $V_{n}=V_{\max }$, the tangent $\left(\Delta_{p}\right)$ becomes horizontal and then $p=0$, which means that

$$
I_{\max }-V_{\max }\left(\frac{\beta h-\beta \xi I_{\max }-\left(\beta V_{\max } / R_{\mathrm{pt}}\right)+\left(1 / R_{\mathrm{pt}}\right)}{\xi+\beta R_{\mathrm{st}} h-\beta R_{\mathrm{st}} \xi I_{\max }-\left(\beta R_{\mathrm{st}} V_{\max } / R_{\mathrm{pt}}\right)}\right)=0 .
$$

As $I_{\max }=V_{\max } / R_{\mathrm{GL}}$, (22) can easily be rearranged to lead to the result of (8).

One can finally notice that approximations (13) and (20) do not affect the analytical nature of the solution, since $i$ and $v$ are assumed infinitely small.

\section{Proposed MPPT Algorithm}

In PV systems, the MPPT controller is aimed at computing $V_{\max }$ as a reference voltage and translating it into a PWM command signal to act on the DC/DC converter and adjust the PV voltage to the computed reference voltage value under varying load and weather conditions. The proposed algorithm is based on $V_{\max }$ computed according to (8) and implemented using the following steps at every sampling time:

(i) measure $I, V$, G, and $T$;

(ii) compute $R_{\mathrm{GL}}=V / I$;

(iii) compute $h=\left(I_{\mathrm{ph}}+I_{0}\right) n_{\mathrm{p}}$;

(iv) compute $V_{\max }$ with (8);

(v) compute $e=V-V_{\max }$ and use it as a PI controller input, for example, to generate the command signal which is translated into PWM form to control the DC/DC converter (Figure 3).

By analyzing the steps of this algorithm, the reference voltage $V_{\max }$ is calculated using algebraic formula (7), once the radiation and temperature are measured. This lets us to think that the convergence of the PV voltage towards $V_{\max }$ will not present any oscillations around the latter. Moreover, the convergence time will be mainly linked to the performance of the used controller to eliminate the error between these two voltages. During our tests, we opted for a PI controller for its simplicity. As mentioned before, the accuracy of $V_{\max }$ is limited by the accuracy of the used model, from which formula (8) was deduced. On the other hand, from (8), one can expect that for any variation of irradiation and/or temperature, an appropriate $V_{\max }$ should be generated. Thus, the MPPT algorithm will be able to adapt to climatic conditions. The calculation time will not be considered as an influence factor, since the algorithm does not require any data storage nor a heavy data processing.

\section{Implementation of the Method}

As mentioned previously, the accuracy of the method is based on the accuracy of the PV model. The mathematical
TABLE 1: SR-20 PV device specifications (A.M.1.5, $1 \mathrm{~kW} / \mathrm{m}^{2}, 25^{\circ} \mathrm{C}$ ).

\begin{tabular}{lc}
\hline Voltage at MPP & $V_{\max }=17.2 \mathrm{~V}$ \\
Current at MPP & $I_{\max }=1.17 \mathrm{~A}$ \\
Short-circuit current & $I_{\mathrm{scr}}=1.28 \mathrm{~A}$ \\
Open circuit voltage & $V_{\mathrm{oc}}=21.6 \mathrm{~V}$ \\
Maximum power & $P_{\max }=20 \mathrm{~W}$ \\
Ideality factor & $A=1.9$ \\
Charge of 1 electron & $q=1.6 \mathrm{e}^{-19} \mathrm{C}$ \\
Boltzmann constant & $1.380658 \mathrm{e}^{-23} \mathrm{~J} / \mathrm{K}$ \\
Band gap energy & $E_{\mathrm{g}}=1.12 \mathrm{eV}$ \\
Reverse saturation current at $T_{\mathrm{r}}$ & $I_{\mathrm{or}}=5.98 \mathrm{e}^{-6} \mathrm{~A}$ \\
Short-circuit current generated at $T_{\mathrm{r}}$ & $I_{\mathrm{scr}}=1.28 \mathrm{~A}$ \\
Temperature coefficient of & $k_{i}=512.10^{-6} \mathrm{~A} / \mathrm{K}$ \\
short-circuit current & $n_{\mathrm{s}}=36$ \\
Number of cells connected in series & $n_{\mathrm{p}}=1$ \\
Number of cells connected in parallel & $R_{\mathrm{s}}=0.004 \Omega$ \\
Internal series resistance of a cell & $R_{\mathrm{p}}=1000 \Omega$ \\
Internal parallel resistance of a cell &
\end{tabular}

model of the PV device should then be validated. The PV device we have used is an SR-20 with the specifications in Table 1.

5.1. PV Model Validation. According to the nonlinearity nature of the algebraic equations (6) and (7), we have used the well-known Newton-Raphson numerical method and Matlab software to implement the model. The simulated $I$ $V$ and $P-V$ characteristics at nominal conditions are shown in Figure 6.

The result shows that the model is able to reproduce the values of the key parameters of the SR-20 PV device, under nominal conditions, such as $V_{\mathrm{oc}}, I_{\mathrm{scr}}, V_{\max }, I_{\max }$, and $P_{\max }$. The model was also validated offline with actual data measured during cloudy periods. The measured irradiance, temperature, and voltage of the panel were stored in a data file to be used as model inputs. The validation test consisted of comparing the measured current with the current provided by the model combined with Newton-Raphson numerical method. The results are shown in the first part of Figure 7. The corresponding measured irradiation, voltage, and temperature are shown in the second, third, and fourth part of Figure 7, respectively.

We can observe that the cloudy periods allowed us to cover a large irradiation interval, between about $200 \mathrm{~W} / \mathrm{m}^{2}$ and more than $800 \mathrm{~W} / \mathrm{m}^{2}$. Under this condition, the current provided by the model followed accurately the measured current. The model could then be considered as valid and robust to determine $V_{\max }$ with (8).

5.2. Experimental Setup. The schematic representation of the experimental platform is given in Figure 8. The proposed study has been implemented using a dSpace 1104 board which works with Matlab/Simulink software. It consists of a PV module SR-20 connected to a resistive variable load. The LEM LA55-P current sensor, the LEM LV20-P voltage 


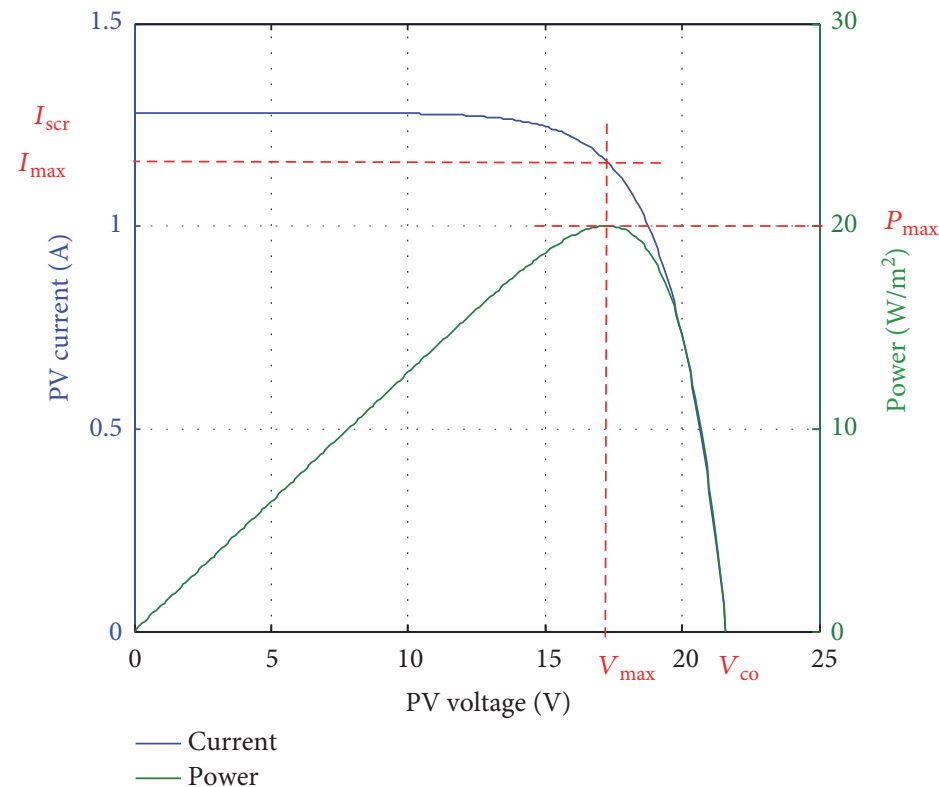

Figure 6: Simulated $I-V$ and $P-V$ characteristics of PV device at $1000 \mathrm{~W} / \mathrm{m}^{2}, T=25^{\circ} \mathrm{C}$.
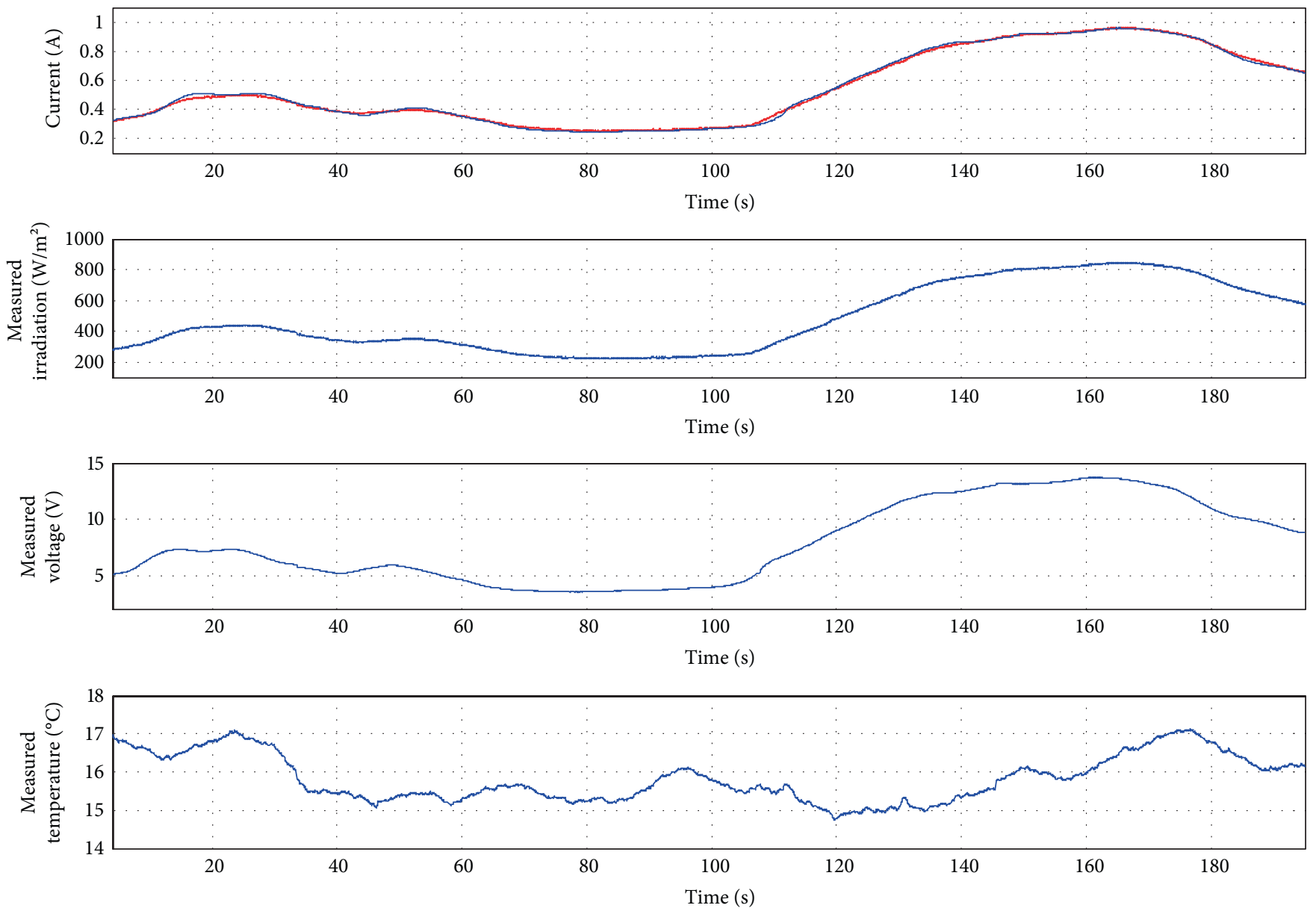

- Measured Estimated

FIGURE 7: Offline validation of the model under natural conditions. 


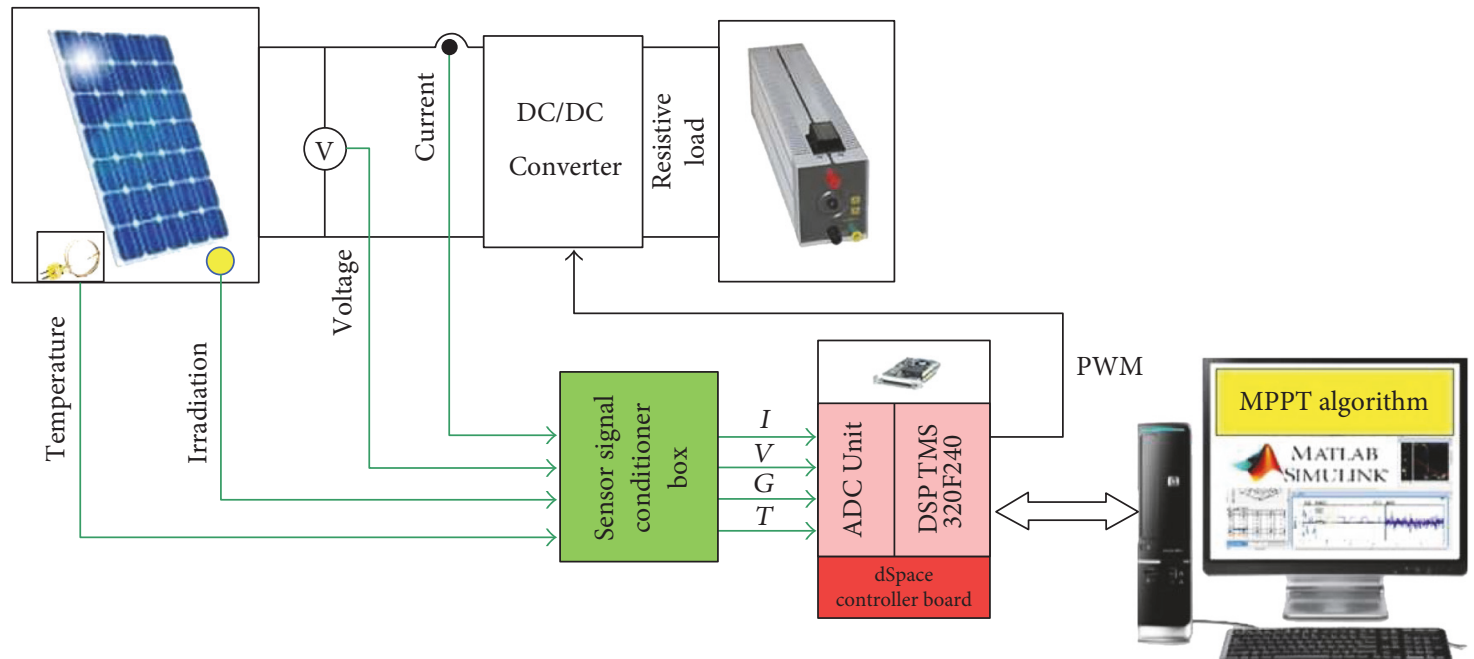

FIGURE 8: Schematic representation of the experimental platform.

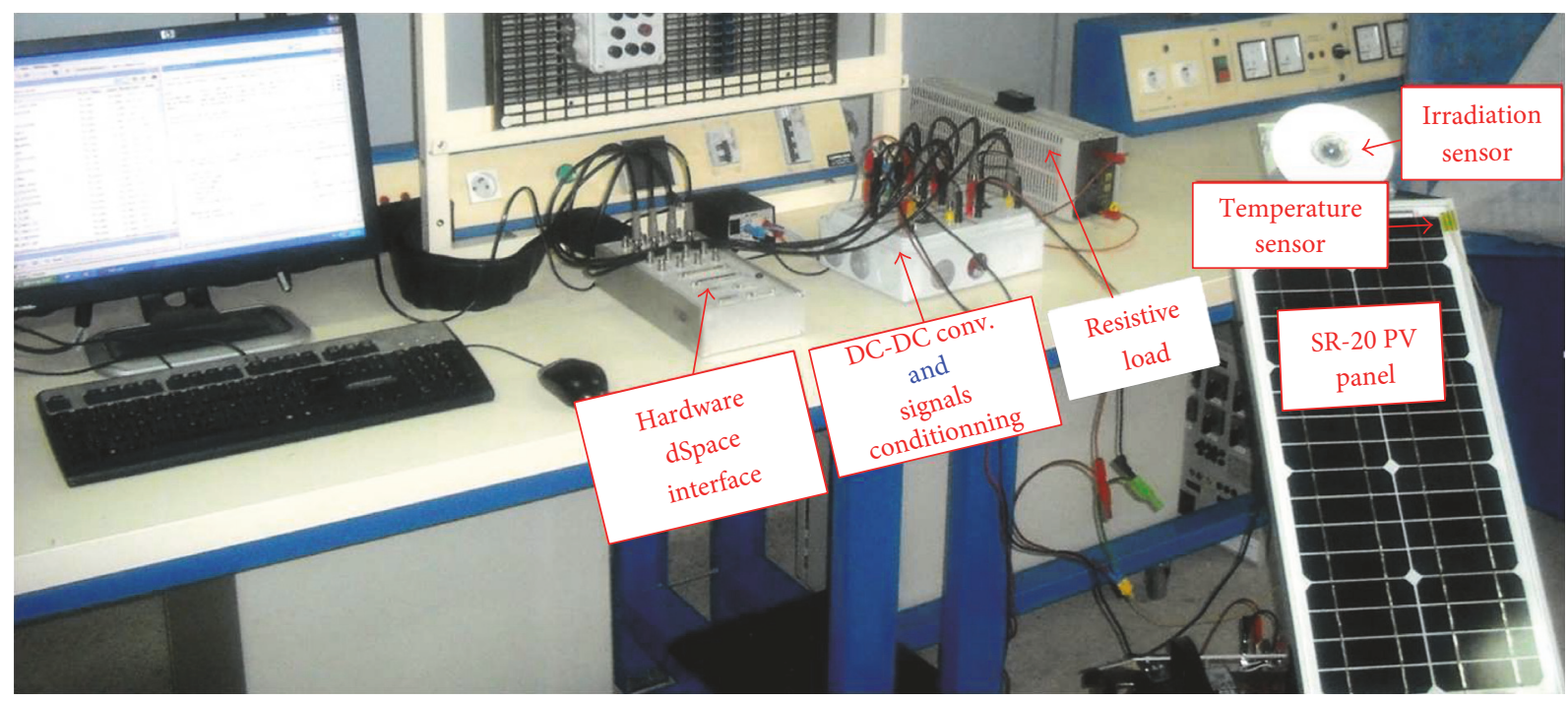

FIGURE 9: Photograph of the experimental setup.

sensor, and the thermocouple temperature sensor $(K)$ are used to measure the current, the voltage, and the temperature of PV module, respectively. All measured signals are conditioned and transformed in the voltage range 0 to \pm 10 volts and sent to the dSpace Controller Board. The latter includes an analog-digital converter (ADC), a digital-analog converter (DAC), and a processing system. The dSpace works on Matlab/Simulink platform which is common engineering software. The dSpace boards are associated with Control Desk software, which makes the data acquisition and realtime analysis easy. The dSpace Real-Time Interface (RTI) is based on the Matlab Real-Time Workshop (RTW) to create real-time codes.

The MPPT algorithm is based on a C-S function code which allows a real-time compilation via a Simulink box. The real-time code is loaded on the dSpace board. A realtime handling of data becomes then easily accessible via the Control Desk software. During the experimental validation, the noisy measured signals are eliminated using a low-pass filter. Figure 9 is a photograph of the designed experimental platform.

5.3. Experimental Results and Discussion. Experimental tests were carried out during alternating sunny and cloudy periods, in Marrakech on June 10, 2016, from $11 \mathrm{~h} 30$ to 18h30. The PV panel we have used was fixed on a mechanical solar tracker. The same tests could also be done with a fixed panel. Figure 10 shows the measured irradiance and temperature. The cloudy periods were observed at the end of the day.

Very large and abrupt irradiation changes, caused by the cloudy periods, gave rise to a high irradiation variation up to almost $700 \mathrm{~W} / \mathrm{m}^{2}$. Which is a favourable condition to test the robustness of the algorithm. 

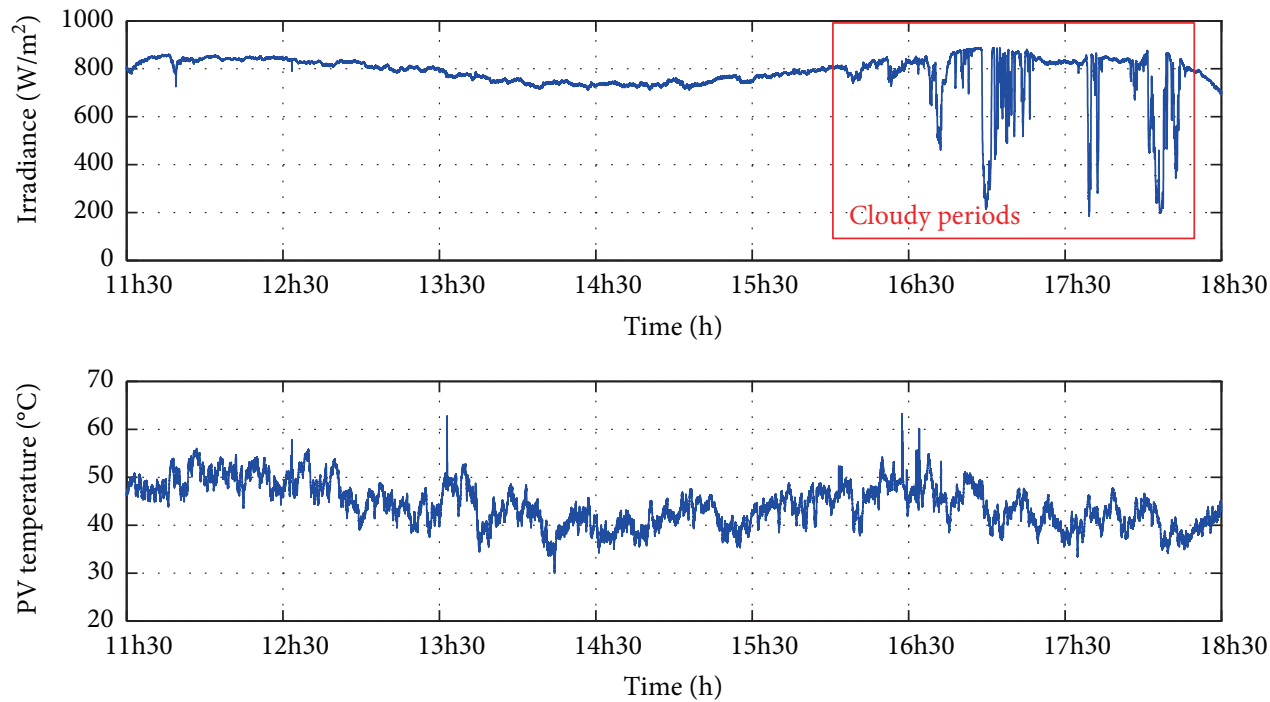

Figure 10: Measured irradiance and temperature in Marrakech on June 10, 2016, from 11h30 to $18 \mathrm{~h} 30$.

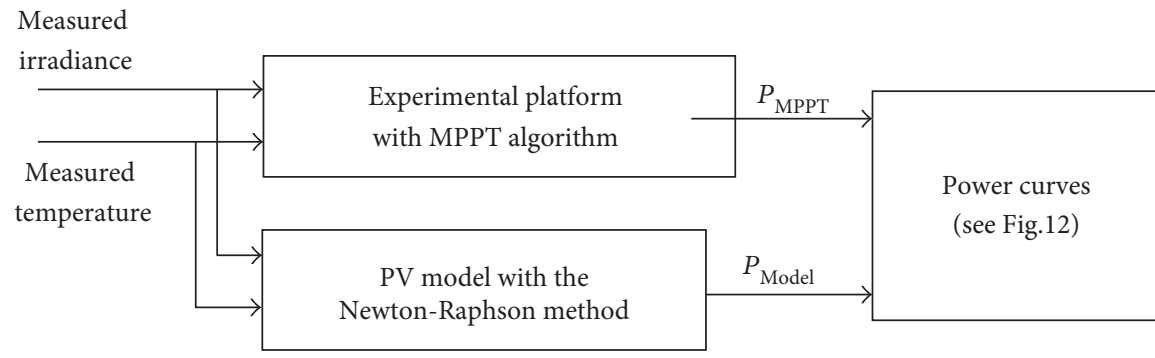

FIGURE 11: Comparison technique of the actual power with the expected one.

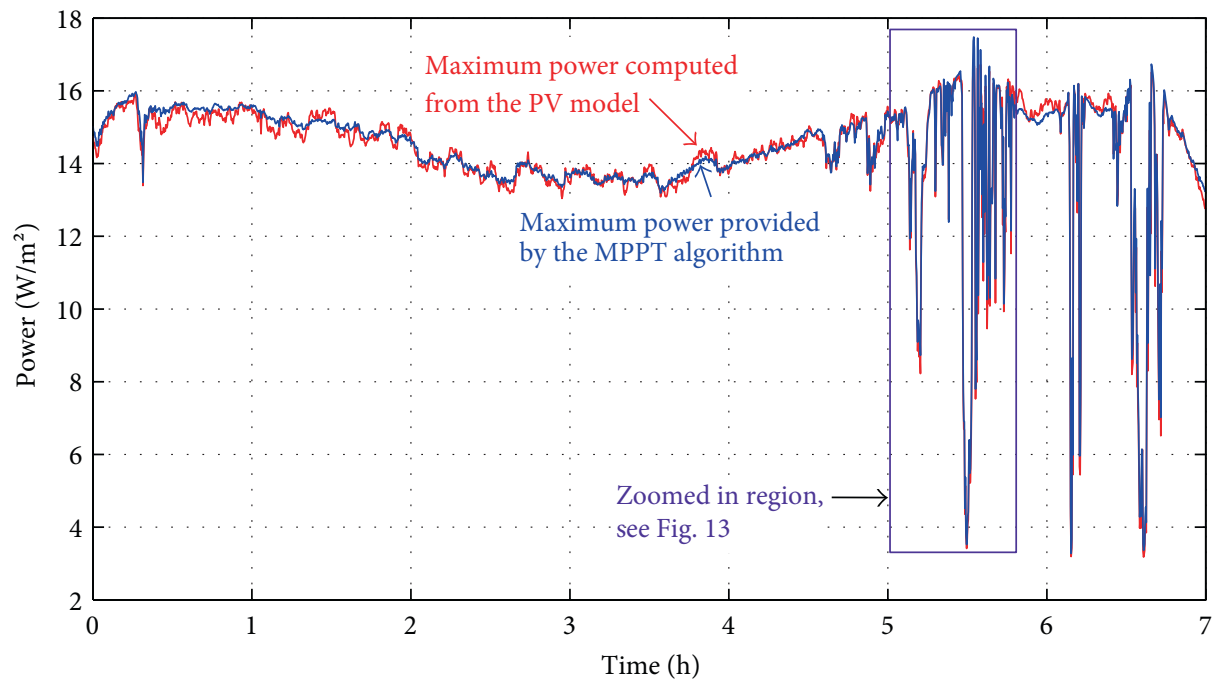

FIGURE 12: Results of the actual power, given by MPPT algorithm, and the power computed using the PV model.

As stipulated in subsection 5.1, the used PV model, combined with the numerical Newton-Raphson method, was able to reproduce with accuracy the behaviour of the PV panel.
The potential maximum power computed from this model was then taken as a reference value. The comparison of the maximum power provided by the proposed MPPT algorithm 


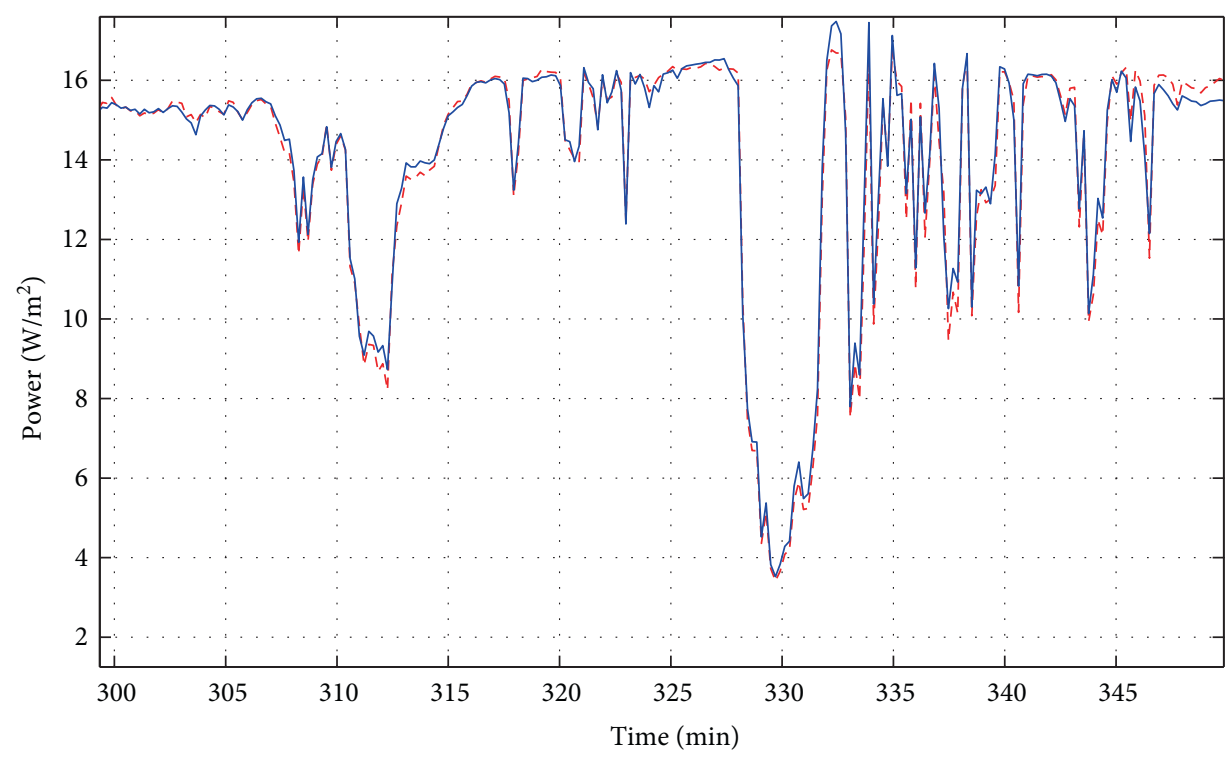

FIgURE 13: Zoom in of the framed part region in Figure 12.
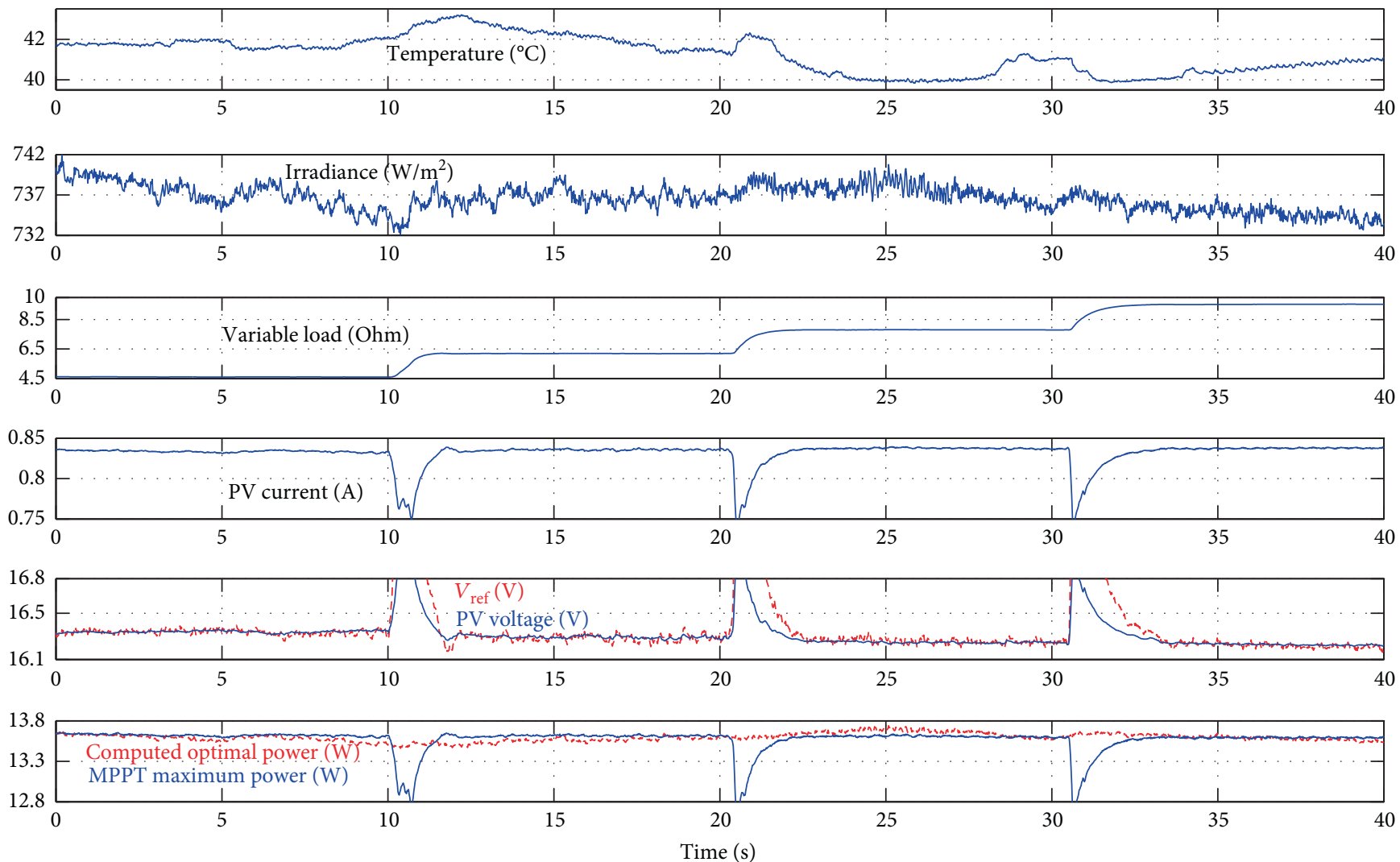

Figure 14: MPPT with a resistive variable load.

with this reference value, according to the diagram of Figure 11, is illustrated in Figure 12.

To closely examine the ability of the method to follow the irradiation fluctuations, the framed part in the cloudy period region of Figure 12 is zoomed in Figure 13.
As can be observed, the proposed MPPT algorithm remains fast and robust in extreme changes of irradiation, especially with the abrupt decrease down to $200 \mathrm{~W} / \mathrm{m}^{2}$.

Since the performance of a PV generator is influenced by the load to which it is connected, it is interesting to test 
if the MPPT algorithm is able to maintain the power at its maximum value in spite of load variations. To do so, we have varied the resistive load according to the profile shown in the third part of Figure 14, within a time interval in which the irradiation was relatively constant.

In the last part of Figure 14, the power has indeed been maintained at its maximum value after every short transient (about $3 \mathrm{~s}$ ) due to abrupt load changes and then the PV current. The observed insignificant accuracy errors are due to PV model accuracy and the acquisition system including cables.

\section{Conclusion}

The effectiveness of the proposed method has been tested in practice with an experimental platform under realistic conditions, such as cloudy periods and a variable resistive load. The obtained results respond to all expectations formulated in the introduction and in the sections where the principle of the method was introduced.

The MPPT algorithm managed to follow rapidly the variations of the optimum power under a variable resistive load and in the case of abrupt and very significant changes of irradiation. The observed insignificant accuracy errors are due to PV model parameters' tuning and the acquisition system including cables. The last kinds of errors could be eliminated by designing an MPPT controller based on a printed circuit board with a specific DC/DC converter and a microcontroller as a calculator.

However, in addition to current and voltage measurements, the PV temperature and irradiance measurements are also needed. The PV model, on which the method is based, must also be accurate. To reduce instrumentation and maintenance costs, a software estimator of the solar irradiation that we developed in our previous publication could be used [18]. Other implications and limitations should be reported. Indeed, our method has not been tested particularly in the case of large PV areas and PV arrays under partial shading and under certain types of cloud cover. This could be an extension of our future works on this subject.

\section{Conflicts of Interest}

The authors declare that they have no conflicts of interest.

\section{References}

[1] N. A. Kelly and T. L. Gibson, "Improved photovoltaic energy output for cloudy conditions with a solar tracking system," Solar Energy, vol. 83, no. 11, pp. 2092-2102, 2009.

[2] A. Zegaoui, M. Aillerie, P. Petit, J. P. Sawicki, J. P. Charles, and A. W. Belarbi, "Dynamic behaviour of PV generator trackers under irradiation and temperature changes," Solar Energy, vol. 85, no. 11, pp. 2953-2964, 2011.

[3] M. A. Eltawil and Z. Zhao, "MPPT techniques for photovoltaic applications," Renewable and Sustainable Energy Reviews, vol. 25, pp. 793-813, 2013.

[4] D. P. Hohm and M. E. Ropp, "Comparative study of maximum power point tracking algorithms," Progress in Photovoltaics: Research and Applications, vol. 11, no. 1, pp. 47-62, 2003.
[5] P. Bhatnagar and R. K. Nema, "Maximum power point tracking control techniques: state-of-the-art in photovoltaic applications," Renewable and Sustainable Energy Reviews, vol. 23, pp. 224-241, 2013.

[6] Z. Salam, J. Ahmed, and B. S. Merugu, "The application of soft computing methods for MPPT of PV system: a technological and status review," Applied Energy, vol. 107, pp. 135-148, 2013.

[7] N. Femia, G. Petrone, G. Spagnuolo, and M. Vitelli, "Optimization of perturb and observe maximum power point tracking method," IEEE Transactions on Power Electronics, vol. 20, no. 4, pp. 963-973, 2005.

[8] F. Liu, S. Duan, F. Liu, B. Liu, and Y. Kang, "A variable step size INC MPPT method for PV systems," IEEE Transactions on Industrial Electronics, vol. 55, no. 7, pp. 2622-2628, 2008.

[9] N. Femia, G. Petrone, G. Spagnuolo, and M. Vitelli, "A technique for improving $\mathrm{P} \& \mathrm{O}$ MPPT performances of doublestage grid-connected photovoltaic systems," IEEE Transactions on Industrial Electronics, vol. 56, no. 11, pp. 4473-4482, 2009.

[10] K. S. Tey and S. Mekhilef, "ScienceDirect modified incremental conductance MPPT algorithm to mitigate inaccurate responses under fast-changing solar irradiation level," Solar Energy, vol. 101, pp. 333-342, 2014.

[11] N. S. D’Souza, L. A. C. Lopes, and X. Liu, "Comparative study of variable size perturbation and observation maximum power point trackers for PV systems," Electric Power Systems Research, vol. 80, no. 3, pp. 296-305, 2010.

[12] Q. Mei, S. Member, M. Shan et al., "A novel improved variable step-size method for PV systems," vol. 58, no. 6, pp. 24272434, 2011.

[13] K. K. Kumar, R. Bhaskar, and H. Koti, "Implementation of MPPT algorithm for solar photovoltaic cell by comparing short-circuit method and incremental conductance method," Procedia Technology, vol. 12, pp. 705-715, 2014.

[14] T. Esram and P. L. Chapman, "Comparison of photovoltaic array maximum power point tracking techniques," vol. 22, no. 2, pp. 439-449, 2007.

[15] F. Chekired, C. Larbes, D. Rekioua, and F. Haddad, "Implementation of a MPPT fuzzy controller for photovoltaic systems on FPGA circuit," Energy Procedia, vol. 6, pp. 541549, 2011.

[16] O. Guenounou, B. Dahhou, and F. Chabour, "Adaptive fuzzy controller based MPPT for photovoltaic systems," Energy Conversion and Management, vol. 78, pp. 843-850, 2014.

[17] M. G. Villalva, J. R. Gazoli, and E. R. Filho, "Comprehensive approach to modeling and simulation of photovoltaic arrays," IEEE Transactions on Power Electronics, vol. 24, no. 5, pp. 1198-1208, 2009.

[18] T. Hassboun, L. E. Bahir, Y. Aite Driss, and M. E. Adnani, "Solar irradiation estimator based on a self-calibrated reference solar cell," Turkish Journal of Electrical Engineering and Computer Sciences, vol. 24, pp. 4885-4899, 2016. 

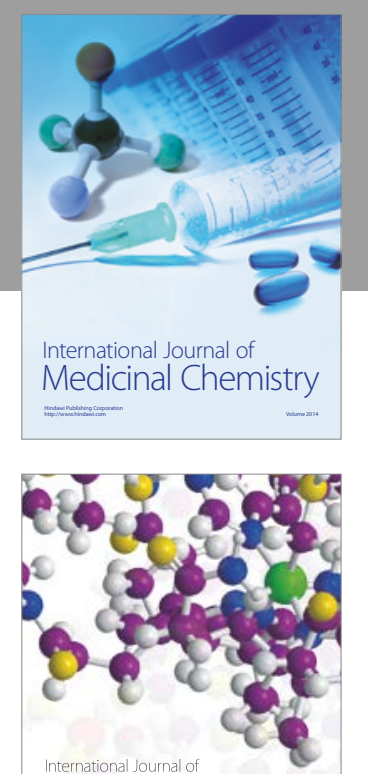

Carbohydrate Chemistry

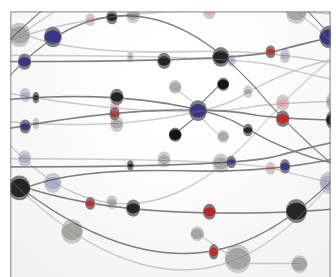

The Scientific World Journal
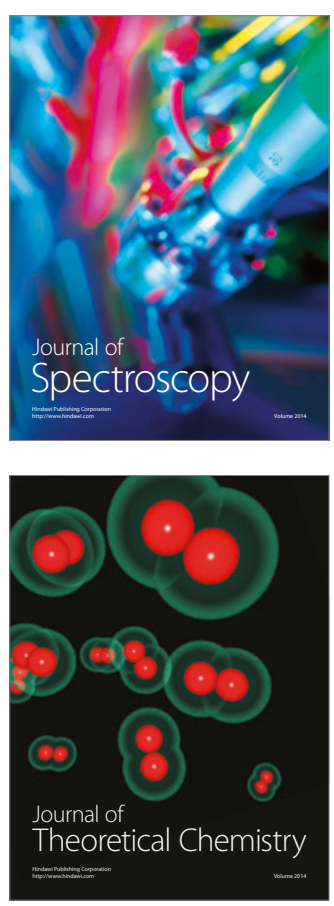
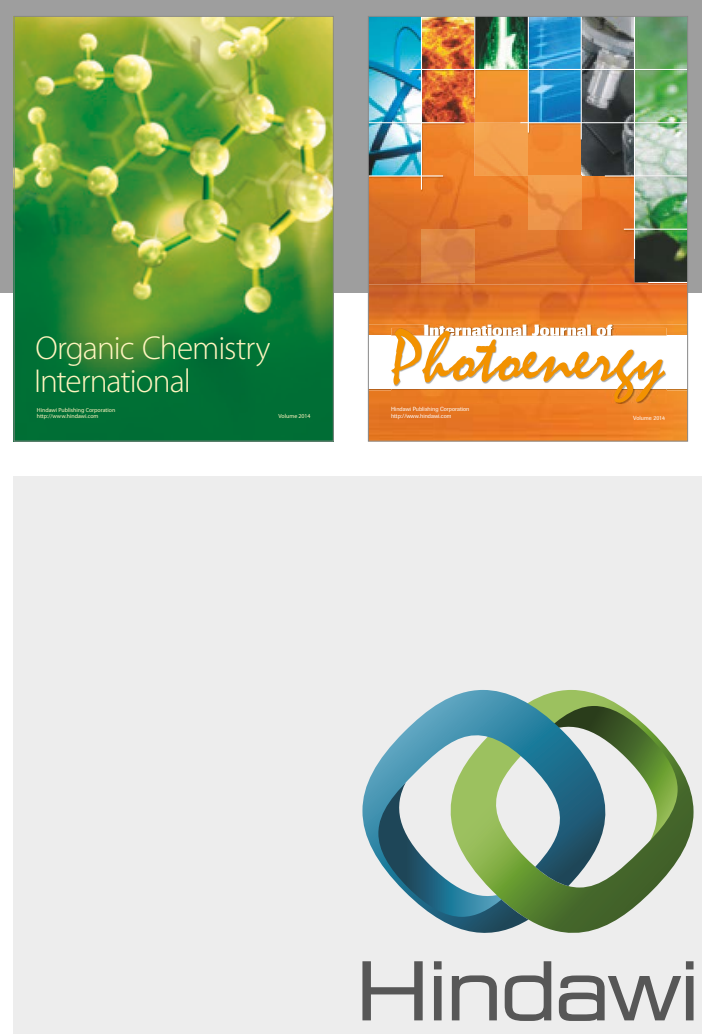

Submit your manuscripts at

https://www.hindawi.com

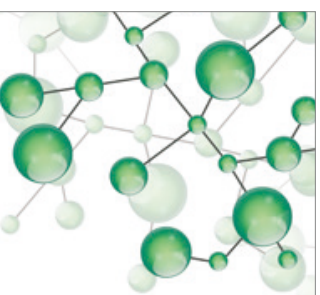

International Journal of

Inorganic Chemistry

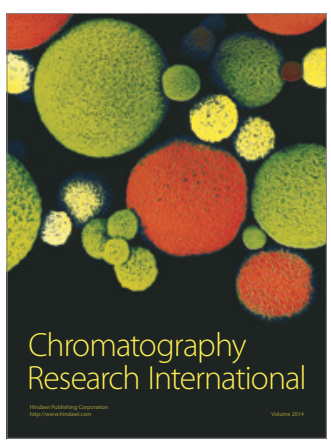

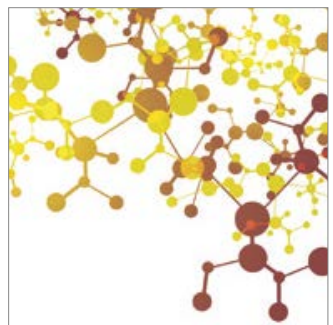

Applied Chemistry
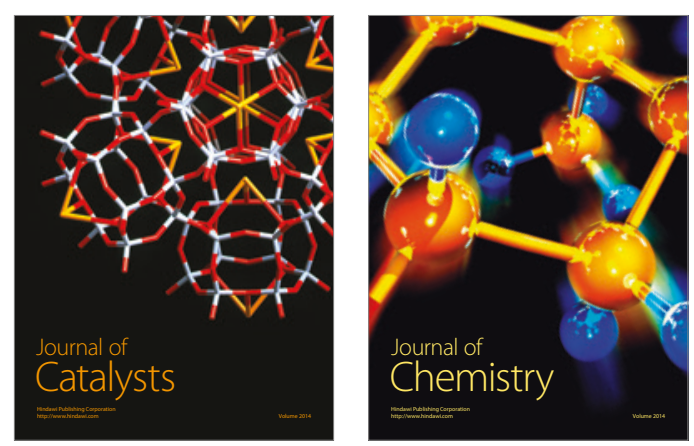
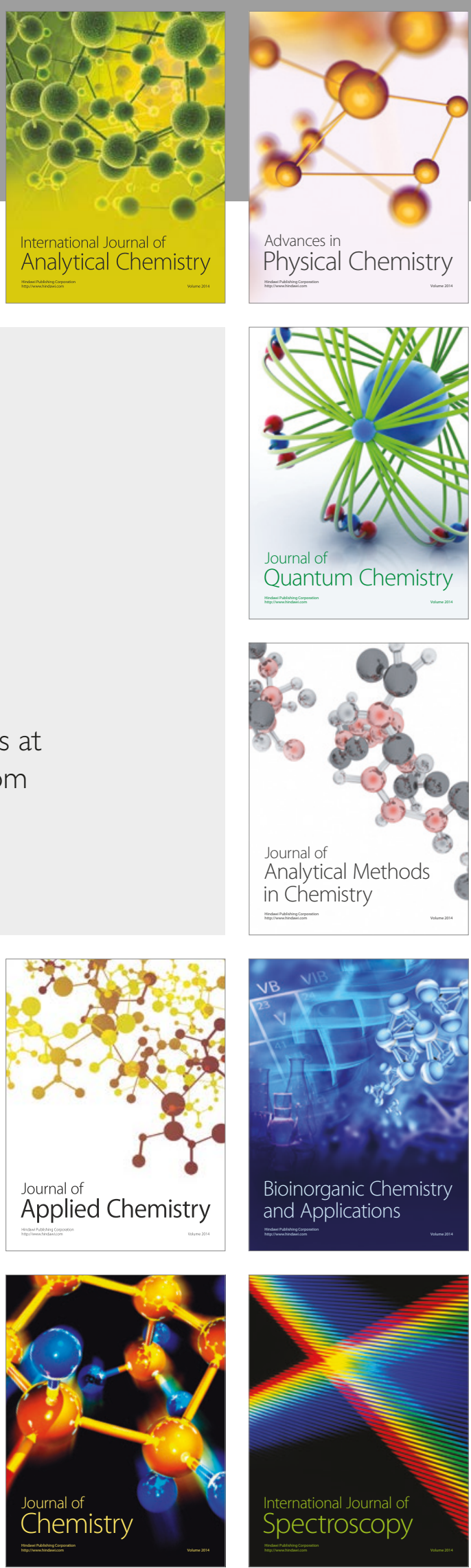